\title{
Estimating Discriminatory Power with Non-normality and a Small Number of Defaults
}

\author{
C. S. Hong ${ }^{1}$ · H. J. Kim² ${ }^{2}$ J. L. Lee ${ }^{3}$ \\ ${ }^{1}$ Department of Statistics, Sungkyunkwan University \\ ${ }^{2}$ Research Institute of Applied Statistics, Sungkyunkwan University \\ ${ }^{3}$ Department of Statistics, Sungkyunkwan University
}

(Received September 4, 2012; Revised September 26, 2012; Accepted October 8, 2012)

\begin{abstract}
For credit evaluation models, we extend the study of discriminatory power based on AUC obtained from a ROC curve when the number of defaults is small and distribution functions of the defaults and non-defaults are normal distributions. Since distribution functions do not satisfy normality in real world, the distribution functions of the defaults and non-defaults are assumed as normal mixture distributions based on results that the normal mixture could be better fitted than other distribution estimation methods for non-normal data. By using several AUC statistics, the discriminatory power under such a circumstance is explored and compared with those of normal distributions.
\end{abstract}

Keywords: AUC, bootstrap, credit evaluation, kernel density, ROC.

\section{1. 서론}

신용평가(credit evaluation) 관점으로 연구하기 위하여 두 종류의 확률변수를 고려한다. 첫번째 확률 변수 $S$ 는 대출기관에서 차주의 신용가치를 예상하기 위해 차주에게 부여한 신용점수(연속형) 또는 신 용등급(이산형)으로 나타내는 스코어 변수이며, 두번째 확률변수 $Z$ 는 차주(borrower)들의 지불능력 상 태(state of solvency)를 $d$ (default; 부도)와 $n$ (non-default; 정상)으로 나타내는 평가변수이다. 스코어 변수 $S$ 를 통한 대출기관의 의도는 차주의 신용가치정보에 의거하여 차주의 미래상태 $Z$ 를 예상하는 것 이다 (Koh, 1992; Sobehart와 Keenan, 2001; Tasche, 2006). 평가변수 $Z$ 는 부도상태 $\{Z=d\}$ 와 정 상상태 $\{Z=n\}$ 일 때로 설정한다. 그리고 주어진 평가변수 $Z$ 가 $d$ 와 $n$ 으로 주어진 스코어 변수 $S$ 의 조 건부 분포함수를 부도차주와 정상차주의 누적분포함수인 $F_{d}(s)$ 와 $F_{n}(s)$ 로 각각 정의할 때, 스코어 $S$ 의 분포함수는 다음과 같이 나타낼 수 있다 (Stein, 2005; Hong과 Choi, 2009; Hong 등, 2010).

$$
F(s)=\lambda F_{d}(s)+(1-\lambda) F_{n}(s),
$$

여기서 $\lambda$ 는 전체부도확률(total probability of default)이다. 즉 $\lambda=P[Z=d]$ 이다.

\footnotetext{
${ }^{1}$ Corresponding author: Professor, Department of Statistics, Sungkyunkwan University, 25-2, Sungkyun
} kwan-ro, Jongro-gu, Seoul 110-745, Korea. E-mail: cshong@skku.edu 
일 년 또는 그 이하의 짧은 기간 동안의 부도율 또는 평가시스템의 판별력을 추정하는 경우와 신용 등급 별 조건부 부도율을 추정하는 경우에 Tasche (2009)는 전체부도확률 $\lambda$ 가 적을 때에 판별력에 대한 추정 문제가 발생한다고 하였다.

Tasche (2009)의 연구 방법을 간략히 요약하면 다음과 같다. 모형 (1.1)을 $F_{d}(s)=\Phi\left(s: \mu_{d}=\right.$ $\left.6.4, \sigma_{d}=1.96\right), F_{n}(s)=\Phi\left(s: \mu_{n}=8, \sigma_{n}=2\right)$ 와 같이 정규분포를 따른다고 가정하고, 이 분포로 부터 독립적인 표본을 추출한다. 전체부도확률이 적은 경우를 고려하기 위하여 표본수를 Table 2.2 에 서 Tasche model인 경우와 동일하게 부도와 정상상태의 표본수 $N_{d}, N_{n}$ 을 각각 추출한다. 분류모형을 시각화하고 성과를 평가하는 유용한 방법으로 $\mathrm{ROC}$ 곡선을 사용하고, 모형의 성과 또는 변별력을 측 정하기 위하여 $\mathrm{AUC}$ (area under curve) 통계량을 이용하였다. 추출한 표본으로부터 커널밀도함수 추 정에 기반한 $\mathrm{AUC}$ 추정량, 정규커널(normal kernel) 밀도함수를 사용한 $\mathrm{AUC}_{\text {Kernel }}$ 와 경험적분포함 수에 기반한 $\mathrm{AUC}$ 추정량 $\mathrm{AUC}_{e m p}$ 값을 계산하고, $\mathrm{AUC}_{e m p}$ 의 정규근사 신뢰구간을 구한다. 부트스트 랩 방법으로 부도와 정상의 표본으로부터 복원 추출로 999 번을 반복하여 표본추출하여, $\mathrm{AUC}_{K e r n e l}$ 와 $\mathrm{AUC}_{e m p}$ 의 부트스트랩 신뢰구간을 각각 구한다. 이 과정을 100 회 반복 시행한 후, 가정된 분포로부터 구한 $\mathrm{AUC}$ 값이 100 개의 $\mathrm{AUC}_{e m p}$ 의 정규근사 신뢰구간 그리고 $\mathrm{AUC}_{K e r n e l}$ 와 $\mathrm{AUC}_{e m p}$ 의 신뢰구간에 어느 정도 포함되는지 확인하였다(AUC에 관한 참고문헌은 Berry와 Linoff (2009)을 참조).

본 연구에서는 모형 $(1.1)$ 의 $F_{d}(s)$ 와 $F_{n}(s)$ 를 정규분포 $\Phi(\cdot)$ 로 가정하고 부도수가 적을 경우에 판별력 을 추정하는 Tasche (2009)의 연구를 확장하고자 한다. 현실 세계의 신용평가 연구에서는 부도와 정상 차주의 누적분포함수 $F_{d}(s)$ 와 $F_{n}(s)$ 가 정규분포를 따르지 않기 때문에 이에 적합한 분포 추정에 많은 연구가 진행되었다. 특히 Hong과 Lee (2011) 그리고 Hong 등 (2011)의 연구에서는 정규혼합(normal mixture) 분포를 이용한 ROC 곡선이 자료에 가장 잘 적합함을 보였다. 따라서 모형 (1.1)의 $F_{d}(s)$ 와 $F_{n}(s)$ 를 비정규(non-normal distribution) 분포인 정규혼합분포로 다음과 같이 가정한다.

$$
\begin{aligned}
& F_{d}(s)=\lambda_{1} \Phi_{d 1}(s)+\left(1-\lambda_{1}\right) \Phi_{d 2}(s), \\
& F_{n}(s)=\lambda_{2} \Phi_{n 1}(s)+\left(1-\lambda_{2}\right) \Phi_{n 2}(s),
\end{aligned}
$$

여기서 $\lambda_{1}, \lambda_{2}$ 는 정규혼합분의 가중값이다.

대부분의 현실 자료에서 $F_{d}(s)$ 와 $F_{n}(s)$ 는 정규분포를 따르지 않으며 모형 (1.1)과 같은 단봉모형보다 는 모형 (1.2)와 같은 이봉모형(bimodal)인 형태이다. 본 연구에서는 이봉형태의 모형 (1.2)로부터 추 출한 표본자료와 표본자료를 단봉형태인 모형 (1.1)로 간주하여 Tasche (2009)의 연구방법을 사용하여 $\mathrm{AUC}$ 값이 100 개의 $\mathrm{AUC}_{e m p}$ 의 정규근사 신뢰구간 그리고 $\mathrm{AUC}_{K e r n e l}$ 와 $\mathrm{AUC}_{e m p}$ 의 신뢰구간에 어느 정도 포함되는지 확인하면서 판별력 추정을 비교 분석하고자 한다.

본 논문의 구성은 다음과 같다. 2 절에서는 Tasche (2009)의 모형을 단봉모형으로 간주하고 이를 이봉모 형으로 확장하여 정규혼합모형을 다양하게 설정한다. 정규혼합에 대응하는 단봉모형의 정규분포도 같 이 설정하였다. 그리고 부도수가 적은 경우를 고려하기 위하여 부도와 정상상태 각각의 표본크기도 설 정한다. 3 절에서는 각각의 모형에 대해 커널밀도함수 추정 및 경험적 분포함수 추정을 이용한 판별력 추정에 대한 결과를 정리하고 비교 분석한다. 마지막으로 4 절에서는 결론을 유도한다.

\section{2. 모형설정}

Tasche (2009)의 모형은 각각의 신용점수가 이산형인 이항분포(binomial distribution)를 따르고 16 개 의 신용등급이 있는 신용평가 모형에서 부도확률을 $p_{d}=0.4$, 정상확률을 $p_{n}=0.5$ 라 가정한다. 이때 
Table 2.1. Model distributions

\begin{tabular}{|c|c|c|c|c|}
\hline Model & UNI/MIX & $d / n$ & Distribution & $\mathrm{AUC}$ \\
\hline \multirow{2}{*}{ Tasche } & \multirow{2}{*}{ Uni } & 부도 & $N\left(6.4,1.96^{2}\right)$ & \multirow{2}{*}{0.7162} \\
\hline & & 정상 & $N\left(8,2^{2}\right)$ & \\
\hline \multirow{4}{*}{1} & \multirow{2}{*}{ Uni } & 부도 & $N\left(5.76,1.39^{2}\right)$ & \multirow{2}{*}{0.9258} \\
\hline & & 정상 & $N\left(8.64,1.43^{2}\right)$ & \\
\hline & \multirow{2}{*}{ Mix } & 부도 & $0.6 N\left(6.4,1.96^{2}\right)+0.4 N\left(4.8,1.83^{2}\right)$ & \multirow{2}{*}{0.8325} \\
\hline & & 정상 & $0.6 N\left(8,2^{2}\right)+0.4 N\left(9.6,1.96^{2}\right)$ & \\
\hline \multirow{4}{*}{2} & \multirow{2}{*}{ Uni } & 부도 & $N\left(5.92,1.48^{2}\right)$ & \multirow{2}{*}{0.8865} \\
\hline & & 정상 & $N\left(8.48,1.53^{2}\right)$ & \\
\hline & \multirow{2}{*}{ Mix } & 부도 & $0.7 N\left(6.4,1.96^{2}\right)+0.3 N\left(4.8,1.83^{2}\right)$ & \multirow{2}{*}{0.8082} \\
\hline & & 정상 & $0.7 N\left(8,2^{2}\right)+0.3 N\left(9.6,1.96^{2}\right)$ & \\
\hline \multirow{4}{*}{3} & \multirow{2}{*}{ Uni } & 부도 & $N\left(6.08,1.4^{2}\right)$ & \multirow{2}{*}{0.8649} \\
\hline & & 정상 & $N\left(8.32,1.47^{2}\right)$ & \\
\hline & \multirow{2}{*}{ Mix } & 부도 & $0.6 N\left(6.4,1.96^{2}\right)+0.4 N\left(5.6,1.91^{2}\right)$ & \multirow{2}{*}{0.7853} \\
\hline & & 정상 & $0.6 N\left(8,2^{2}\right)+0.4 N\left(8.8,1.99^{2}\right)$ & \\
\hline
\end{tabular}

Table 2.2. Sample sizes of models

\begin{tabular}{c|ccccccccccc}
\hline Model & \multicolumn{7}{c}{$N_{d}$ or $\left(N_{d 1}, N_{d 2}\right)$} & $N_{n}$ or $\left(N_{n 1}, N_{n 2}\right)$ \\
\hline Tasche & 5 & 10 & 15 & 20 & 25 & 30 & 35 & 40 & 45 & 50 & 250 \\
\hline 1,3 & $(3,2)$ & $(6,4)$ & $(9,6)$ & $(12,8)$ & $(15,10)$ & $(18,12)$ & $(21,14)$ & $(24,16)$ & $(27,18)$ & $(30,20)$ & $(150,100)$ \\
2 & $(4,1)$ & $(7,3)$ & $(11,4)$ & $(14,6)$ & $(18,7)$ & $(21,9)$ & $(25,10)$ & $(28,12)$ & $(32,13)$ & $(35,15)$ & $(175,75)$ \\
\hline
\end{tabular}

부도와 정상 스코어를 연속형 정규분포로 근사시키기 위하여 이항분포 $b(16,0.4)$ 와 $b(16,0.5)$ 를 이용하 여 다음과 같이 각각의 모수를 유도하였다.

$$
F_{d}(s) \equiv N\left(\mu_{d}=6.4, \sigma_{d}^{2}=1.96^{2}\right), \quad F_{n}(s) \equiv N\left(\mu_{n}=8, \sigma_{n}^{2}=2^{2}\right) .
$$

그리고 이에 대응하는 $\mathrm{AUC}$ 통계량값은 $\Phi\left(\left(\mu_{n}-\mu_{d}\right) / \sqrt{\sigma_{n}^{2}+\sigma_{d}^{2}}\right)$ 식을 이용하여 0.7162이다 (Hanley와 McNeil, 1982).

다음 Model 1, 2, 3은 부도와 정상을 단일정규분포(uni normal)라 가정하는 단일(Uni)모형과 정규혼합 분포(normal mixture)라 가정하는 혼합(Mix)모형으로 구분한다.

먼저 식 (1.2)에서 정규혼합분포의 가중값인 $\lambda_{1}=0.6$ 와 이항분포 $b(16,0.4), b(16,0.3)$ 를 이용하여 $F_{d}(s) \equiv 0.6 N\left(6.4,1.96^{2}\right)+0.4 N\left(4.8,1.83^{2}\right)$ 를 설정하고, $\lambda_{2}=0.6$ 와 이항분포 $b(16,0.5), b(16,0.6)$ 를 이용하여 $F_{n}(s) \equiv 0.6 N\left(8,2^{2}\right)+0.4 N\left(9.6,1.96^{2}\right)$ 를 설정하여 Model 1: Mix로 한다. 또한 Model 1: $\mathrm{Mix}$ 의 혼합분포로부터 1 차와 2 차 적률을 구하여 $F_{d}(s) \equiv N\left(5.76,1.39^{2}\right)$ 과 $F_{n}(s) \equiv N\left(8.64,1.43^{2}\right)$ 의 단일모형으로 설정하고 이를 Model 1: Uni로 하였다. Model 2: Mix는 Model 1: Mix에서 가 중값 $\lambda_{1}, \lambda_{2}=0.7$ 로 변경하여 설정하였고, 이 혼합분포로부터 Model 2: Uni를 설정한다. 마지 막으로 Model 3: Mix는 Model 1: Mix에서 이항분포를 수정하여 $b(16,0.4), b(16,0.35)$ 를 이용 하여 $F_{d}(s) \equiv 0.6 N\left(6.4,1.96^{2}\right)+0.4 N\left(5.6,1.91^{2}\right)$ 를 설정하고, $b(16,0.5), b(16,0.55)$ 를 이용하여 $F_{n}(s) \equiv 0.6 N\left(8,2^{2}\right)+0.4 N\left(8.8,1.99^{2}\right)$ 를 설정한다. Model 3: Uni는 Model 3: Mix의 혼합분포 로부터 1 차와 2 차 적률을 구하여 단일모형으로 설정한다. Table 2.1 에 이 모형들을 정리하였다.

본 연구에서는 부도수가 적은 경우를 고려하기 위하여 부도와 정상상태 각각의 표본크기를 Table 2.2에 정하였고 총 부도수는 Tasche (2009)와 동일하게 설정하여 동일한 부도율의 상태에서 연구한다. 
Table 3.1. Model 1: Uni

\begin{tabular}{cccccccc}
\hline \multirow{2}{*}{$N_{d}$} & \multicolumn{3}{c}{ AUC in interval } & & \multicolumn{3}{c}{$50 \%$ in interval } \\
\cline { 2 - 3 } \cline { 6 - 7 } & Kernel & emp. & normal & & Kernel & emp. & normal \\
\hline 5 & 69 & 68 & 77 & & 0 & 0 & 1 \\
10 & 71 & 72 & & & 0 & 0 & 0 \\
15 & 83 & 85 & 87 & & 0 & 0 & 0 \\
20 & 85 & 84 & 88 & & 0 & 0 & 0 \\
25 & 89 & 89 & 92 & & 0 & 0 & 0 \\
30 & 84 & 84 & 88 & & 0 & 0 & 0 \\
35 & 88 & 87 & 90 & & 0 & 0 & 0 \\
40 & 91 & 92 & 90 & & 0 & 0 & 0 \\
45 & 90 & 91 & 93 & & 0 & 0 & 0 \\
50 & 93 & 93 & 94 & & 0 & 0 & 0 \\
\hline
\end{tabular}

Table 3.2. Model 1: Mix

\begin{tabular}{|c|c|c|c|c|c|c|}
\hline \multirow{2}{*}{$N_{d}$} & \multicolumn{3}{|c|}{ AUC in interval } & \multicolumn{3}{|c|}{$50 \%$ in interval } \\
\hline & Kernel & emp. & normal & Kernel & emp. & normal \\
\hline 5 & 79 & 77 & 83 & 6 & 7 & 19 \\
\hline 10 & 81 & 82 & 82 & 0 & 0 & 0 \\
\hline 15 & 88 & 88 & 91 & 0 & 0 & 0 \\
\hline 20 & 90 & 90 & 92 & 0 & 0 & 0 \\
\hline 25 & 92 & 92 & 92 & 0 & 0 & 0 \\
\hline 30 & 93 & 93 & 94 & 0 & 0 & 0 \\
\hline 35 & 93 & 93 & 95 & 0 & 0 & 0 \\
\hline 40 & 95 & 94 & 97 & 0 & 0 & 0 \\
\hline 45 & 91 & 93 & 93 & 0 & 0 & 0 \\
\hline 50 & 94 & 93 & 96 & 0 & 0 & 0 \\
\hline
\end{tabular}

\section{3. 판별력 분석}

1절에서 간략히 소개한 Tasche (2009)와 동일한 연구 방법으로 Model 1, Model 2, Model 3에 대하 여 부트스트랩 표본추출을 100 번 반복 시행하여 100 개의 부트스트랩 신뢰구간을 생성하고 이 100 개의 $95 \%$ 신뢰구간 안에 AUC값이 어느 정도 포함되는지를 비교하여 본다. Table 3.1은 Model 1: Uni의 모 의실험 결과이다.

Model 1: Uni에서의 AUC값은 0.9258이며, Table 3.1을 통하여 신뢰구간에 포함되는 $\mathrm{AUC}$ 값이 포 함되는 정도('AUC in interval')가 세 종류의 신뢰구간이 모두 90 이 넘는 부도수는 40 개 이상이였 다. 또한 $\mathrm{AUC}$ 의 신뢰구간에서 의미없는 무작위 모형(random model)을 의미하는 0.5 가 포함되는 정 도('50\% in interval')를 살펴보면, 부도수가 10 개 이상부터는 포함하지 않는다. 경험적분포 신뢰구 간('emp')과 커널추정 신뢰구간('Kernel')은 모든 다른 크기의 부도수에 대하여 AUC의 포함 비율의 차이가 거의 없다. 그러나 부도수가 35 이하일 경우에는 정규근사 신뢰구간('normal')이 경험적분포 신 뢰구간과 커널추정의 신뢰구간보다 좋으며, 부도수가 40 이상인 경우에는 세 가지 신뢰구간의 성능의 차이는 없어진다. 따라서 Tasche (2009)의 연구 결과와 비교해 보면, Model 1: Uni의 AUC는 Tasche (2009) 연구의 AUC값 0.7162 보다 0.2096 커진 값이며, 'AUC in interval'과 ' $50 \%$ in interval'은 Tasche (2009)연구보다 적어진 것을 파악할 수 있다.

Model 1: Mix에서의 AUC값은 0.8325이며, Model 1: Uni보다 0.0933 작다. Table 3.2의 'AUC in 
Table 3.3. Model 2: Uni

\begin{tabular}{|c|c|c|c|c|c|c|}
\hline \multirow{2}{*}{$N_{d}$} & \multicolumn{3}{|c|}{ AUC in interval } & \multicolumn{3}{|c|}{$50 \%$ in interval } \\
\hline & Kernel & emp. & normal & Kernel & emp. & normal \\
\hline 5 & 67 & 68 & 75 & 2 & 3 & 4 \\
\hline 10 & 82 & 84 & 87 & 0 & 0 & 0 \\
\hline 15 & 81 & 83 & 84 & 0 & 0 & 0 \\
\hline 20 & 85 & 87 & 90 & 0 & 0 & 0 \\
\hline 25 & 87 & 86 & 91 & 0 & 0 & 0 \\
\hline 30 & 91 & 91 & 94 & 0 & 0 & 0 \\
\hline 35 & 90 & 91 & 92 & 0 & 0 & 0 \\
\hline 40 & 90 & 90 & 91 & 0 & 0 & 0 \\
\hline 45 & 93 & 95 & 94 & 0 & 0 & 0 \\
\hline 50 & 91 & 92 & 94 & 0 & 0 & 0 \\
\hline
\end{tabular}

Table 3.4. Model 2: Mix

\begin{tabular}{|c|c|c|c|c|c|c|}
\hline \multirow{2}{*}{$N_{d}$} & \multicolumn{3}{|c|}{ AUC in interval } & \multicolumn{3}{|c|}{$50 \%$ in interval } \\
\hline & Kernel & emp. & normal & Kernel & emp. & normal \\
\hline 5 & 79 & 79 & 83 & 18 & 16 & 29 \\
\hline 10 & 84 & 87 & 89 & 1 & 1 & 3 \\
\hline 15 & 90 & 90 & 95 & 1 & 1 & 1 \\
\hline 20 & 92 & 92 & 94 & 1 & 1 & 1 \\
\hline 25 & 96 & 95 & 96 & 0 & 0 & 0 \\
\hline 30 & 92 & 92 & 95 & 0 & 0 & 0 \\
\hline 35 & 96 & 97 & 97 & 0 & 0 & 0 \\
\hline 40 & 97 & 97 & 98 & 0 & 0 & 0 \\
\hline 45 & 96 & 96 & 97 & 0 & 0 & 0 \\
\hline 50 & 96 & 96 & 98 & 0 & 0 & 0 \\
\hline
\end{tabular}

interval'은 Model 1: Uni보다 많다. 특히 부도수가 적을 때 훨씬 좋은 것을 알 수 있다. 하지만 부도 수가 20 개 이상인 경우부터는 세 가지 신뢰구간 모두 90 이상 포함한다. ‘ $50 \%$ in interval'은 부도수 가 10 개 이상부터는 포함하고 있지 않다. 그리고 부도수가 5 개인 경우, Model 1: Uni보다 Model 1: $\operatorname{Mix}$ 가 더 많음을 알 수 있다. 부도수가 15 이하일 경우에는 정규근사 신뢰구간이 경험적분포 신뢰구간 과 커널추정의 신뢰구간보다 좋으며, 부도수가 20 개 이상인 경우에는 세 신뢰구간의 성능의 차이는 없 다. 이것은 부도와 정상분포를 각각 정규혼합으로 가정했을 때, $\mathrm{AUC}$ 값은 단일 정규분포로 가정했을 때 보다 작아지지만, $\mathrm{AUC}$ 값의 추정에 있어서는 정규혼합분포 가정에서의 커널과 경험적분포 함수추정이 좋다고 판단한다.

Model 2: Uni의 AUC값은 0.8865이며, Model 1: Uni보다 0.0393 작은 값이다. Table 3.3의 'AUC in interval'을 살펴보면, 부도수가 30 개 이상인 경우부터는 세가지 신뢰구간 모두 90 이상 포함하며 부 도수가 10 개 이상인 경우에는 Model 1: Uni보다 많이 포함한다. ' $50 \%$ in interval'은 부도수가 5 개 이 하일 때 많이 포함되고 부도수가 5개인 경우에는 Model 1: Uni보다는 조금 많음을 알 수 있다. 경험적 분포 신뢰구간과 커널추정 신뢰구간은 모든 다른 크기의 부도수에 대하여 $\mathrm{AUC}$ 의 포함 비율의 차이가 거의 없다. 그러나 부도수가 25 이하일 경우에는 정규근사 신뢰구간이 경험적분포 신뢰구간과 커널추정 의 신뢰구간보다 좋으며, 부도수가 30 이상인 경우에는 세 신뢰구간의 성능의 차이는 없어진다. 그러므 로 Model 2: Uni의 AUC값은 Model 1: Uni보다 작으나 AUC 포함비율은 높다고 탐색한다.

Model 2: Mix에서의 AUC값은 0.8082이며, Model 2: Uni보다 0.0783 작고 Model 1: Mix보다는 
Table 3.5. Model 3: Uni

\begin{tabular}{|c|c|c|c|c|c|c|}
\hline \multirow{2}{*}{$N_{d}$} & \multicolumn{3}{|c|}{ AUC in interval } & \multicolumn{3}{|c|}{$50 \%$ in interval } \\
\hline & Kernel & emp. & normal & Kernel & emp. & normal \\
\hline 5 & 67 & 67 & 73 & 5 & 7 & 1 \\
\hline 10 & 75 & 76 & 80 & 0 & 0 & 0 \\
\hline 15 & 83 & 83 & 85 & 0 & 0 & 0 \\
\hline 20 & 89 & 89 & 92 & 0 & 0 & 0 \\
\hline 25 & 85 & 85 & 89 & 0 & 0 & 0 \\
\hline 30 & 89 & 89 & 92 & 0 & 0 & 0 \\
\hline 35 & 93 & 94 & 96 & 0 & 0 & 0 \\
\hline 40 & 90 & 90 & 90 & 0 & 0 & 0 \\
\hline 45 & 91 & 92 & 92 & 0 & 0 & 0 \\
\hline 50 & 92 & 93 & 94 & 0 & 0 & 0 \\
\hline
\end{tabular}

Table 3.6. Model 3: Mix

\begin{tabular}{|c|c|c|c|c|c|c|}
\hline \multirow{2}{*}{$N_{d}$} & \multicolumn{3}{|c|}{ AUC in interval } & \multicolumn{3}{|c|}{$50 \%$ in interval } \\
\hline & Kernel & emp. & normal & Kernel & emp. & normal \\
\hline 5 & 79 & 79 & 83 & 14 & 16 & 24 \\
\hline 10 & 81 & 82 & 87 & 4 & 5 & 6 \\
\hline 15 & 92 & 92 & 92 & 0 & 0 & 0 \\
\hline 20 & 94 & 93 & 95 & 0 & 0 & 0 \\
\hline 25 & 92 & 92 & 92 & 0 & 0 & 0 \\
\hline 30 & 94 & 93 & 96 & 0 & 0 & 0 \\
\hline 35 & 91 & 92 & 95 & 0 & 0 & 0 \\
\hline 40 & 93 & 92 & 94 & 0 & 0 & 0 \\
\hline 45 & 95 & 94 & 96 & 0 & 0 & 0 \\
\hline 50 & 92 & 93 & 94 & 0 & 0 & 0 \\
\hline
\end{tabular}

0.0243 크다. Table 3.4의 'AUC in interval'을 보면 부도수가 작을 때 Model 2: Uni보다 훨씬 좋은 것을 알 수 있지만 부도수가 15 개 이상인 경우부터는 포함비율이 세 가지 신뢰구간 모두 90 이상으로 Model 1: Mix와 Model 2: Mix는 모두 전반적으로 좋다. ' $50 \%$ in interval'은 부도수가 25개 이상부 터는 포함하지 않으며 부도수가 적을 때 Model 2: Mix는 Model 2: Uni와 Model 1: Mix보다 포함 비 율이 훨씬 많다. 부도수가 15 이하일 경우에는 정규근사 신뢰구간이 경험적분포 신뢰구간과 커널추정 의 신뢰구간보다 좋으며, 부도수가 20 개 이상인 경우에는 세 신뢰구간의 성능의 차이는 없어진다. 그러 므로 정규혼합모형에서의 $\mathrm{AUC}$ 값은 단일 정규분포로 가정했을 때보다 작지만, $\mathrm{AUC}$ 값의 추정에 있어 서는 정규혼합분포 가정에서의 커널과 경험적분포함수 추정이 좋다고 판단할 수 있다. 그리고 정규혼합 가정에서는 Model 1: Mix보다는 Model 2: Mix의 AUC값이 작고 'AUC in interval'은 전반적으로 좋 지만 ‘ $50 \%$ in interval’도 많아진 것을 파악한다.

Model 3: Uni의 AUC값은 0.8649이며, Model 1: Uni와 유사함을 알 수 있다. Table 3.5의 'AUC in interval'에서 부도수가 35개 이상일 때 세가지 신뢰구간 모두 90이상 포함하며, Model 1: Uni와 비슷 하다. ' $50 \%$ in interval'을 살펴보면, 부도수가 5 개 이하일 때 많이 포함되는데, Model 1: Uni보다는 부도수가 5 개인 경우 조금 많다. 부도수가 30 이하일 경우에는 정규근사 신뢰구간이 경험적분포 신뢰구 간과 커널추정의 신뢰구간보다 좋으며, 부도수가 35 이상인 경우에는 세 가지 신뢰구간의 성능의 차이 는 없어진다. Table 3.5를 바탕으로 Model 1: Uni와 비교하면, 'AUC in interval'에서는 차이를 보이 


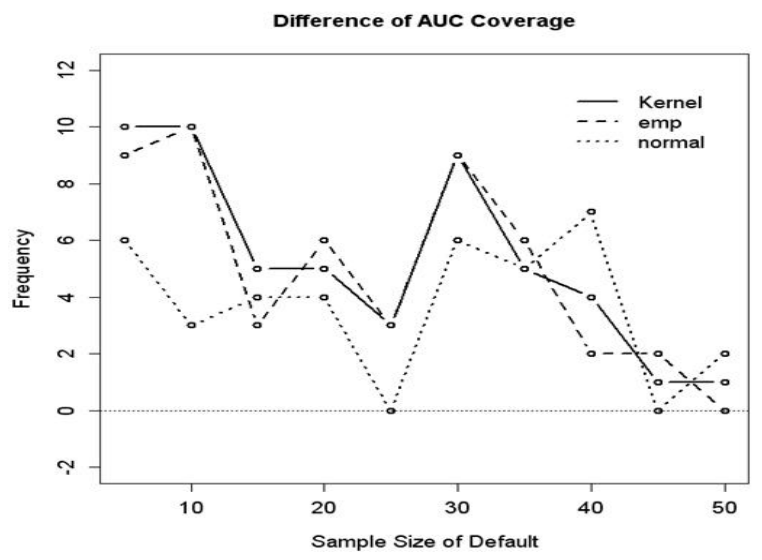

Figure 4.1. Difference in Model 1

지 않았지만, ‘50\% in interval’은 Model 1: Uni보다 많다.

Model 3: Mix의 AUC값은 0.7853이며, Model 3: Uni와 Model 1: Mix보다 조금 작다. Table 3.6의 'AUC in interval'은 Model 3: Uni보다 많이 포함하고 있고 특히 부도수가 적을 때 훨씬 좋지만 부도수 가 15 개 이상인 경우부터는 세 종류의 신뢰구간 모두 $90 \%$ 이상 포함되며, Model 1: Mix와는 거의 유 사함을 파악할 수 있다. ' $50 \%$ in interval'은 부도수가 15 개 이상부터는 포함하고 않으며, 부도수가 적 을 때는 Model 3: Uni와 Model 1: Mix보다 더 많음을 알 수 있다. 부도수가 10개 이하일 경우에는 정 규근사 신뢰구간이 경험적분포 신뢰구간과 커널추정의 신뢰구간보다 좋으나, 부도수가 15 개 이상인 경 우에는 세 신뢰구간의 차이는 없다. 그러므로 정규혼합모형에서의 $\mathrm{AUC}$ 값은 단일 정규분포로 가정했을 때보다 작지만, $\mathrm{AUC}$ 값의 추정에 있어서는 정규혼합분포 가정에서의 커널과 경험적분포함수 추정이 좋 다. 정규혼합 가정에서는 Model 1: Mix보다 Model 3: Mix의 $\mathrm{AUC}$ 값이 작고, $\mathrm{AUC}$ 값이 포함되는 정 도는 유사하지만 ‘ $50 \%$ in interval'은 많아진 것을 발견할 수 있다.

\section{4. 결론}

현실 세계의 신용평가 연구에서는 부도와 정상차주의 누적분포함수가 정규분포를 따르지 않는데도 불구 하고 정규분포를 가정하였다. 본 연구에서는 비정규분포를 따른다고 가정하고 비정규분포를 추정하는 여러 방법 중 정규혼합분포를 이용하였다. 특히 부도수가 적은 경우를 고려하면서 다양한 정규혼합 모 형을 설정하여 이에 대응하는 $\mathrm{ROC}$ 곡선에서의 $\mathrm{AUC}$ 통계량값을 구하고 $\mathrm{AUC}$ 값이 포함되는 비율을 살 펴보았다. 정규혼합분포에 대한 결과를 단일 정규분포로 간주해서 얻은 결과와 비교하였다.

본 연구의 3 절에서 얻은 연구 결과에서 각 Model에서 정규혼합과 단일 정규분포와의 판별력의 차이를 시각적으로 살펴보기 위하여 AUC 포함비율의 차이를 Figure 4.1부터 Figure 4.3에 나타내었다.

Model 1에 대한 Figure 4.1을 살펴보면, 부도수가 증가할수록 차이는 감소한다. 그리고 커널분포함수 로 추정한 $\mathrm{AUC}$ 포함비율의 차이가 가장 크고 정규근사 신뢰구간이 작다. Model 2 와 Model 3 에 대한 Figure 4.2와 Figure 4.3에서도 부도수가 증가할수록 차이는 감소함을 발견할 수 있다. Model 2 에 대한 Figure 4.2 에서는 커널분포함수로 추정한 $\mathrm{AUC}$ 포함비율의 차이가 크고 정규근사 신뢰구간이 작으나, Figure 4.1과 비교하면 분포추정에 따르는 차이가 적다. Model 2 에서의 가중값 $\lambda$ 가 Model 1 보다 증 


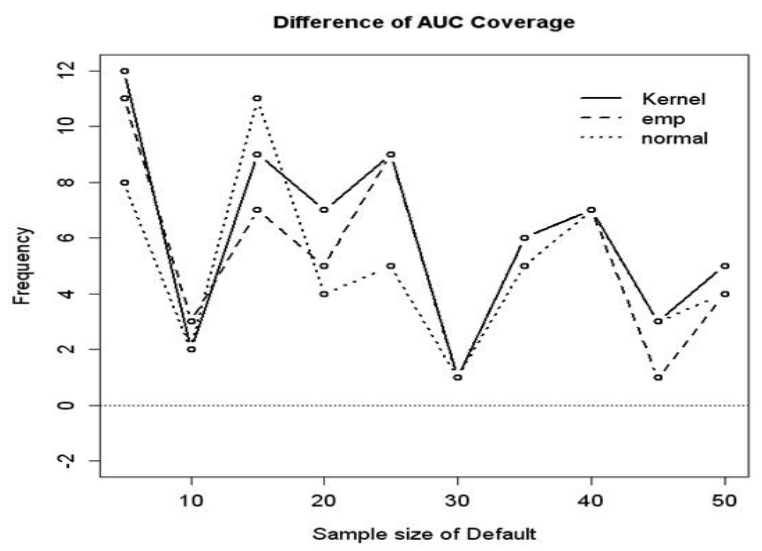

Figure 4.2. Difference in Model 2

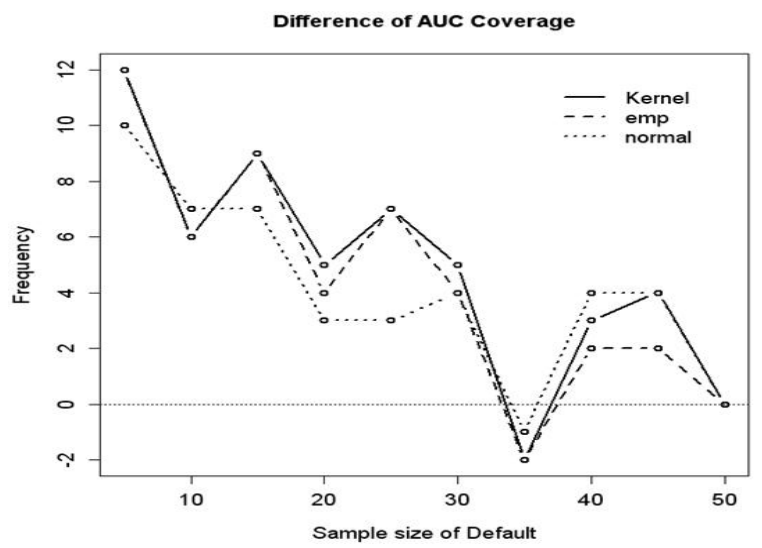

Figure 4.3. Difference in Model 3

가하여 정규혼합의 효과가 감소되었기 때문에 분포추정에 따르는 차이가 적어짐을 발견하였다. Model 3 에 대한 Figure 4.3에서는 AUC 포함비율이 분포추정 종류에 따라 차이가 적음을 Figure 4.1과 비교하 여 파악할 수 있다. Model 3: Mix에서의 평균모수들이 Model 1: Mix보다 가까이 위치함으로 정규혼 합의 효과가 감소되었기 때문에 분포추정에 따르는 차이가 적어짐을 판단할 수 있다.

그러므로 신용평가 자료가 정규성을 만족하지 않고 부도수가 작은 경우에는 정규분포를 가정하는 것보 다 정규혼합분포를 이용하여 부도와 정상분포의 판별력을 추정하는 $\mathrm{AUC}$ 통계량이 좋은 결과를 나타낸 다는 것을 발견하였다. 따라서 본 연구 결과를 활용하면, 부도수가 작고 정규성을 만족하지 않은 경우에 판별력 추정에 효과적이다.

\section{References}

Berry, M. J. A. and Linoff, G. (2009). Data Mining Techniques: For Marketing sales, and Customer Support, Morgan Kaufmann Publishers. 
Hanley, A. and McNeil, B. (1982). The meaning and use of the area under a receiver operating characteristics curve, Diagnostic Radiology, 143, 29-36.

Hong, C. S. and Choi, J. S. (2009). Optimal threshold from ROC and CAP curves, The Korean Journal of Applied Statistics, 11, 911-921.

Hong, C. S., Joo, J. S. and Choi, J. S. (2010). Optimal thresholds from mixture distributions, The Korean Journal of Applied Statistics, 16, 13-28.

Hong, C. S. and Lee, W. Y. (2011). ROC curve fitting with normal mixtures, The Korean Journal of Applied Statistics, 10, 269-278.

Hong, C. S., Lin, M. H. and Hong, S. W. (2011). ROC function estimation, The Korean Journal of Applied Statistics, 8, 987-994.

Koh, H. C. (1992). The Sensitivity of Optimal cutoff to misclassification costs of type I and type II errors in the going concern prediction context, Journal of Business Finance and Accounting, 19, $187-197$.

Sobehart, J. R. and Keenan, S. C. (2001). Measuring default accurately, Credit Risk Special Report, 14, $31-33$.

Stein, R. M. (2005). The relationship between default prediction and lending profits: Integrating ROC analysis and loan pricing, Journal of Banking and Finance, 29, 1213-1236.

Tasche, D. (2006). Validation of internal rating systems and PD estimates, The Analytics of Risk Model Validation, 28, 169-196.

Tasche, D. (2009). Estimating discriminatory power and PD curves when the number of defaults is small, Working Paper, Lloyds Banking Group. 